\title{
Joint Node Deployment and Wireless Energy Transfer Scheduling for Immortal Sensor Networks
}

\author{
Rong Du*, Carlo Fischione*, Ming Xiao ${ }^{\dagger}$ \\ * Department of Network and Systems Engineering, ${ }^{\dagger}$ Communication Theory Department \\ KTH Royal Institute of Technology, Stockholm, Sweden \\ Email: $\{$ rongd, carlofi, mingx $\}$ kth.se
}

\begin{abstract}
The lifetime of a wireless sensor network (WSN) is limited by the lifetime of the individual sensor nodes. A promising technique to extend the lifetime of the nodes is wireless energy transfer. The WSN lifetime can also be extended by exploiting the redundancy in the nodes' deployment, which allows the implementation of duty-cycling mechanisms. In this paper, the joint problem of optimal sensor node deployment and WET scheduling is investigated. Such a problem is formulated as an integer optimization whose solution is challenging due to the binary decision variables and non-linear constraints. To solve the problem, an approach based on two steps is proposed. First, the necessary condition for which the WSN is immortal is established. Based on this result, an algorithm to solve the node deployment problem is developed. Then, the optimal WET scheduling is given by a scheduling algorithm. The WSN is shown to be immortal from a networking point of view, given the optimal deployment and WET scheduling. Theoretical results show that the proposed algorithm achieves the optimal node deployment in terms of the number of deployed nodes. In the simulation, it is shown that the proposed algorithm reduces significantly the number of nodes to deploy compared to a random-based approach. The results also suggest that, under such deployment, the optimal scheduling and WET can make WSNs immortal.
\end{abstract}

\section{INTRODUCTION}

Wireless sensor networks (WSNs) have been investigated for monitoring several scenarios such as forests [1], pipelines [2], and battle-fields [3]. In all these cases, we desire that the WSNs work as long as possible, such that the WSNs had an adequate lifetime, or even had infinite lifetime. However, long or infinite lifetime is hard or impossible to achieve, unless we recharge the nodes' battery.

To endow WSNs with immortality, i.e., infinite network lifetime, the basic idea is to use rechargeable battery and enable the sensor nodes to harvest energy from environment, such as solar [4], wind [5], and vibration sources [6]. However, energy harvesting faces a major problem that the arrival of the ambient energy is hard to predict or even control [7]. Therefore, the performance of energy harvesting may be compromised.

A more appealing solution is given by wireless energy transmission (WET), where the sensor nodes wirelessly receive energy and store it into their rechargeable battery. We can use a base station to transmit radio-frequency energy, while the nodes are equipped with rectifying-antenna (rectena) to harvest the energy and store it in the battery. As we can control the transmission power, time, and direction at the

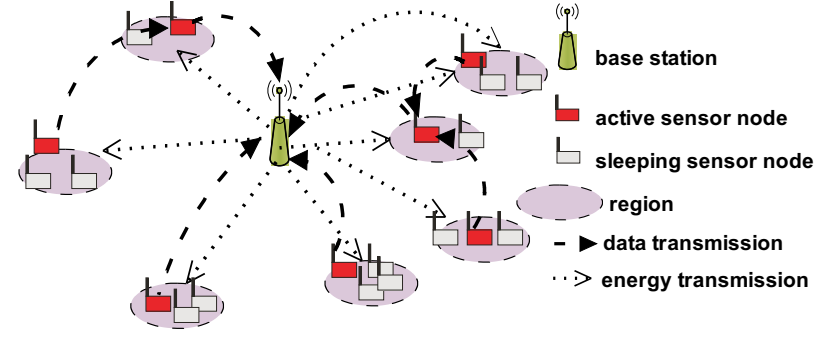

Fig. 1. The wireless energy transfer enabled wireless sensor network considered in the paper

base station, the WET is more controllable than the energy harvesting approaches mentioned above. However, the WET efficiency, which is the ratio of the power that the node receives to the transmission power at the base station, is usually limited due to the distance among base station and nodes, and the low energy conversion rate on the node side. Therefore, we need energy beamforming techniques [8], which can greatly increase the received energy on the node side, and greatly extend nodes' lifetime.

However, when the WSN contains many sensor nodes, the base station may not be able to supply enough energy to each and every node such that we have an immortal WSN. To solve the problem, we consider that nodes deployed at nearby locations can take turns to work to reduce energy consumption. Since these nodes are co-located, they can receive electromagnetic energy at the same time and we say these nodes are in the same small region. Such approach makes it potentially possible that the WSN has infinite network lifetime, and could be applied in the monitoring of scenarios such as agricultural, urban traffic monitoring, and pipeline monitoring. Therefore, we consider a WET enabled WSN system as described in Fig. 1. The WSN consists of one base station and multiple sensor nodes. The base station transmits energy to the nodes and collects the measurements from them, while the nodes receive the electro-magnetic energy from the base station, and measure and transmit data, in duty-cycle, to the base station.

To have the WSN immortal, we need to determine not only how to optimally deploy the sensor nodes, i.e., how many sensor nodes are needed for each region, but also how the base station should schedule the energy transmission to the sensor nodes at each region. Moreover, we need to determine 
the duty cycle of the sensor nodes in each region, according to the WET scheduling at the base station. The contribution of the paper is summarized as follows:

- We propose a novel optimization problem for sensor node deployment and WET scheduling for WET enabled WSNs, which for our best knowledge has never been studied before;

- Given the complexity of the problem and the lack of existing solutions, we propose a novel solution approach based on two steps: 1) greedy based node deployment, 2) WET scheduling based on the deployment results.

- We provide the necessary condition on the sensor node deployment, such that the WSN can be immortal. Based on this, we provide a greedy algorithm and we show that such algorithm achieves the optimal node deployment;

- We further provide the WET scheduling algorithm, such that, under the optimal deployment achieved by the proposed algorithm and a mild requirement on the battery size of sensor nodes, the WSN is immortal.

The rest of the paper is organized as follows. We present the related works in Section II. The WSN system model is presented in Section III, where the necessary condition on node deployment for immortal WSN is established. In Section IV, we provide the algorithm to achieve the optimal sensor node deployment for immortal WSN. Then in Section V, the WET scheduling given the deployment is provided to guarantee the immortality of the WSN. In Section VI, numerical results from simulations are given to show the performance of the proposed algorithm. The paper is concluded in Section VII.

\section{RELATED WORKS}

Network lifetime is an important metric for WSNs. Generally, it is considered as the time till the first node expires its energy [9]. However, recalling that, in each region, multiple nodes are co-located to make measurements in our case, we define the network lifetime as the time till all the sensor nodes in one region expire. It depends not only on the energy consumption of the sensor nodes, but also on their battery size. With the use of rechargeable battery, the battery size of a node could be considered as infinite, if one can charge the node with more energy than it consumes.

Energy harvesting is one possible way to provide energy to sensor nodes [10], since there are various kinds of energy source in our ambient environment [4], [6]. Thus, the nodes can schedule their functioning based on the harvested energy to improve the WSN performance. For example, Michelusi et. al. [11], [12] have proposed that the nodes could transmit more data when they have more incoming harvested energy, such that the utility of the received data at the fusion center is maximized. The problem on the scheduling of the nodes' sensing time such that the WSN sensing utility is maximized has been considered in [13]. They have proposed a randomized myopic policy that selects a group of nodes with the highest energy level to perform sensing. The joint problem on the power control and the placement of the relay node is also considered in [14]. The results showed the significant improvement of jointly optimizing the placement and the power allocation compared to only optimizing only one of them. Considering the case where a sensor transmits all the data in its buffer using all the harvested energy, Ozcelikkale et. al. [15] have provided the bound on the achievable distortion at the fusion center side. Due to that the arrival of the energy is hard to predict and control, the scheduling on nodes is online in practice, which may be worse than the case of off-line optimal scheduling [16].

An alternative solution to charge devices is WET [17], [7], where sensor nodes harvest energy from the electro-magnetic wave. Such idea has been applied to improve network throughput for general networks. In [18], the operations and architectures of simultaneously information and energy transmission are discussed. Wu et. al. [19] have studied the performance of packet transmission of nodes powered by radio-frequency energy. In [20], the joint problem of power control and WET for a relay network has been considered to maximize the achievable rate. WSNs can also benefit from WET in terms of network lifetime. In [21], a charging vehicle has been considered to charge the sensor nodes. Thus, the authors proposed an algorithm to find the path and the charging time of the vehicle. However, the authors assumed that the charging efficiency is large enough, such that the WSN is immortal. Thus, the algorithm may fail when the charging efficiency is not large enough. In [9], the authors have studied the WET scheduling from a base station to WSN, such that the network lifetime is maximized. However, they showed that the WSN is only immortal when the network size is small. Thus, we consider not only the WET scheduling, but also the deployment of co-located nodes in the WSN to reduce the nodes' energy consumption and make the WSN immortal. In our best knowledge, this is the first work on jointly optimization on node deployment and WET scheduling for WET enabled WSN.

\section{SyStem MOdEL AND PROBlem Formulation}

In this section, we first describe the system model of the WSN. Then, we formulate the joint node deployment and WET scheduling problem, and study the necessary condition such that the WSN is immortal under WET from a base station.

We consider a WET enabled WSN as shown in Fig. 1, which consists of one base station and several sensor nodes to monitor an area of interest. The base station is responsible for collecting data from the nodes, and also for transmitting energy wirelessly to the nodes. The nodes are used to measure and relay data to the base station. The nodes are equipped with rechargeable battery and rectenna, which allows them receiving energy from the base station and use the energy later.

We assume that the area to monitor consists of $N$ separated regions, $l_{1}, \ldots, l_{N}$, where $x_{i}$ sensor nodes are deployed in $l_{i}$. The benefit of having multiple nodes in the same region is the robustness against node failures. We wish to determine the optimal $x_{i}$. Time is slotted, and a timeslot consists of 
two phases: energy transmission phase and data transmission phase. Due to that the WET efficiency, i.e., the ratio of the harvested power at a node to the transmission power at the base station, is not high, the base station forms a sharp beam in every timeslot to increase the harvested energy [8] in specific regions of interest. Thus, we assume that all the co-located nodes at a specific region can harvest the electromagnetic wave energy if the base station transmits energy to that region. In the energy transmission phase, all the nodes at that region harvest the energy and store it into their batteries. We normalize the transmission power at the base station to 1 . For a region $l_{i}$, as the nodes have approximately same distance to the base station, we assume that they have approximately the same time-averaged received power. The time-averaged total received power of the nodes in a region depends on a factor, which is denoted by $\alpha_{i}$ and accounts for the path loss, the channel, and the energy conversion loss. We call it region dependent factor. Besides, the received power also depends on the number of nodes that are co-located at that region, and we call it neighbor dependent factor. Such factor is a concave function on the number of nodes in that region, and the intuition is that, the total amount of received energy is sub-additive (due to shadowing of the nodes) on the number of nodes in the region. We use a function $g\left(x_{i}\right)$ to represent such factor, where $x_{i}$ is the number of nodes in the region, and $g\left(x_{i}\right)$ satisfies $0 \leq g^{\prime}\left(x_{i}\right) \leq 1, g^{\prime \prime}\left(x_{i}\right) \leq 0, \forall x_{i} \geq 1, g(1)=1$. Then, the time-averaged WET efficiency of a node in $l_{i}$ is $\alpha_{i} g\left(x_{i}\right) / x_{i}$.

In the data uploading phase, the sensor nodes at the same region take turns to work, i.e., in each timeslot, one of the node is active to measure, to receive data from nodes transmitting to it, and to transmit data to its next node, while the other nodes are sleeping to save energy. Similar to [21], the parameters in the energy consumption model are constant and region dependent. The routing table of each node is fixed in terms of region. That is, if $l_{j}$ is the next hop region of $l_{i}$, then, in a timeslot, the active node in $l_{i}$ will transmit its data to the active node in $l_{j}$. To have a good monitoring of the field in every timeslot, at least one node for every region should be active, and the sampling rate for each region $l_{i}$ should be larger or equal to a threshold $\mu_{i}$. Then, the minimum data rate of the sensor nodes in $l_{i}$ is $\lambda_{i}=\mu_{i}+\sum_{j \in \mathcal{S}(i)} \mu_{j}$, where $\mathcal{S}(i)$ is the set of regions in the sub-tree of the routing tree that is rooted in $l_{i}$. Recalling that the nodes in the same region have the same routing table, we model the time-averaged energy consumption of the nodes to be region dependent. That is, for the nodes in $l_{i}$, the time-averaged energy consumption is $c_{i}\left(\lambda_{i}\right)$ if the node is activated, and it is 0 if it is not activated. Note that the sampling rate requirements $\mu_{i}$ are pre-determined and fixed, $\lambda_{i}$ are also pre-determined and fixed. Thus, $c_{i}\left(\lambda_{i}\right)$ are fixed and we use $c_{i}$ in the following for simplicity ${ }^{1}$. Denote

\footnotetext{
${ }^{1}$ Here, we only consider the consumption in data transmission similar to [21], [22], whereas the circuit consumptions are neglected, and the sleeping node consume no energy for simplicity. However, we should mention that, if circuit consumptions are taken into account, the proposed method still applies by proper modification, which will be one of our future work.
}

$v_{i j}$ the $j$-th node in region $i$, and $E_{i j}(t)$ the residual energy of $v_{i j}$ at $t$. Let $y_{i}(t)=1$ if the base station transmits energy to $l_{i}$ in timeslot $t$, otherwise, $y_{i}(t)=0$. Also, let $z_{i j}(t)=1$ if the $j$-th node in $l_{i}$ is active in $t$. Then, the energy dynamic of the node $v_{i j}$ can be modelled as

$$
E_{i j}(t+1)=\min \left\{E_{i j}(t)+y_{i}(t) \frac{\alpha_{i} g\left(x_{i}\right)}{x_{i}}, B\right\}-z_{i j}(t) c_{i},
$$

where $B$ is the battery buffer of every node. Here, we assume that the battery buffers are large, i.e., $B \gg c_{i}, B \gg 1, \forall i, j$. This is generally true for WSNs, as the nodes are usually designed for a long-term monitoring [23].

Recalling that the base station can transmit energy to only one region in a timeslot, we have that

$$
\sum_{i=1}^{N} y_{i}(t)=1, \forall t
$$

Also, at least one node in a region should be active for a timeslot. Notice that we need to reduce the energy consumptions as much as possible, only one node in a region is active. Thus

$$
\sum_{j=1}^{x_{i}} z_{i j}(t)=1, \forall i, t
$$

To summarize, we can model a WSN monitoring a field by a tuple $(\boldsymbol{L}, \boldsymbol{\lambda}, \boldsymbol{\alpha}, \boldsymbol{x}, \boldsymbol{y}, \boldsymbol{z}, \boldsymbol{E}, B, g)$, where $\boldsymbol{L}=\left\{l_{i}\right\}, \boldsymbol{\lambda}=\left\{\lambda_{i}\right\}$, $\boldsymbol{\alpha}=\left\{\alpha_{i}\right\}, \boldsymbol{x}=\left\{x_{i}\right\}, \boldsymbol{y}=\left\{y_{i}(t) \mid \sum_{k=1}^{N} y_{k}(t)=1, \forall t\right\}$, $\boldsymbol{z}=\left\{z_{i j}(t) \mid \sum_{k=1}^{x_{i}} z_{i k}(t)=1, \forall i, t\right\}$, and $\boldsymbol{E}=\left\{E_{i j}(0) \mid l_{i} \in\right.$ $\left.\boldsymbol{L}, 1 \leq j \leq x_{i}\right\}$. Then, we define the immortality of a WSN as follows:

Definition 1: A WSN $\quad(\boldsymbol{L}, \boldsymbol{\lambda}, \boldsymbol{\alpha}, \boldsymbol{x}, \boldsymbol{y}, \boldsymbol{z}, \boldsymbol{E}, B, g) \quad$ is immortal if and only if, in any timeslot $t$ and any region $l_{i}$, the residual energy of any sensor node is non-negative, i.e., $E_{i j}(t) \geq 0$.

Remark 1: Correspondingly, the lifetime of a WSN $(\boldsymbol{L}, \boldsymbol{\lambda}, \boldsymbol{\alpha}, \boldsymbol{x}, \boldsymbol{E}, B, g)$, with WET-scheduling $\boldsymbol{y}$ and node activation $z$, is defined as the first timeslot $t$ such that $\exists l_{i} \in L, E_{i j}(t+1)<c_{i}, \forall 1 \leq j \leq x_{i}$.

Then, the Joint Node Deployment and WET Scheduling Problem is to find $x_{i}, y_{i}(t)$, and $z_{i j}(t) \forall i, j$, such that the WSN is immortal under WET, as formulated below:

$$
\begin{aligned}
\min _{\boldsymbol{x}, \boldsymbol{y}, \boldsymbol{z}} & \sum_{i=1}^{N} x_{i} \\
\text { s.t. } & E_{i j}(t) \geq 0, \forall i, j, t \\
& \text { Eq. }(1), \forall i, j, t, \text { Eq. (2), } \forall t, \text { Eq. }(3), \forall j, t \\
& x_{i} \in \mathbb{Z}^{+}, y_{i}(t) \in\{0,1\}, z_{i j}(t) \in\{0,1\}, \forall i, j, t
\end{aligned}
$$

where Constraint ( $4 b)$ means that the sensor nodes cannot use more energy than they currently have.

Problem (4) is difficult, not only due to the integer and binary decision variables, but also the indeterministic size of variables (see $z$ as an example). To solve Problem (4), we propose a solution method based on two steps: 1) We find the optimal deployment that satisfies the necessary condition 
of the WSN immortality, and 2) We find the WET scheduling $y_{i}(t)$ and the activation scheduling $z_{i j}(t)$ given the deployment in 1) such that the WSN is immortal.

To begin with, we have the necessary condition such that the WSN is immortal as described in the following lemma.

Lemma 1: Consider a WSN $(\boldsymbol{L}, \boldsymbol{\lambda}, \boldsymbol{\alpha}, \boldsymbol{x}, \boldsymbol{y}, \boldsymbol{z}, \boldsymbol{E}, B, g)$. If it is immortal, then $\sum_{i=1}^{N} c_{i} /\left(\alpha_{i} g\left(x_{i}\right)\right) \leq 1$.

Proof: As the WSN is immortal, there exists a corresponding WET scheduling. Given a large enough duration $T$, we denote $\beta_{i}$ the percentage of timeslots that the base station transfers energy to region $l_{i}$. Then, we have

$$
\sum_{i=1}^{N} \beta_{i}=1 \text {. }
$$

Also, we have that, for each region $l_{i}$, the total energy consumption should be less than or equal to the charged energy. For the total energy consumption, it is $c_{i} T$. For the charged energy, it is $\alpha_{i} g\left(x_{i}\right) \beta_{i} T$. Thus, it has to be that $\alpha_{i} g\left(x_{i}\right) \beta_{i} \geq c_{i}, \forall i$. This gives

$$
\beta_{i} \geq \frac{c_{i}}{\alpha_{i} g\left(x_{i}\right)}, \forall i
$$

We replace $\beta_{i}$ in (5) by (6) and achieve that

$$
\sum_{i=1}^{N} \frac{c_{i}}{\alpha_{i} g\left(x_{i}\right)} \leq 1,
$$

which completes the proof.

Thus, the necessary condition that Problem (4) has feasible solution is given by the following lemma:

Lemma 2: Consider a joint node deployment and WET scheduling Problem (4), the necessary condition that it has feasible solution is

$$
\sum_{i=1}^{N} \lim _{x_{i} \rightarrow+\infty} \frac{c_{i}}{\alpha_{i} g\left(x_{i}\right)}<1 .
$$

The proof is similar to the proof for Lemma 1. Thus, we skip it for saving spaces.

Based on this necessary condition, we determine the deployment of the sensor nodes, as will be discussed in the next section.

\section{NOdE DEPLOYMENT FOR IMMORTAL WSN}

In this section, we will first formulate the deployment problem based on the results from the previous section. Then, we will provide the solution approach to the deployment problem.

\section{A. Node deployment sub-problem}

As discussed in the previous section, even though the nodes in WSN can be charged wirelessly by the base station, the network could still expire due to the low WET efficiency and high energy consumption. To obtain an immortal WSN, we can use the well known idea of applying sleep/awake mechanism. Recalling that $\sum_{i} c_{i} / \alpha_{i} g\left(x_{i}\right) \leq 1$ in Lemma 1 is the necessary condition for the immortality of the WSN, we have that the deployment problem consists in finding how many nodes to deploy in each region, such that this necessary condition is satisfied. This is formalized as follows:

$$
\begin{array}{ll}
\min _{\boldsymbol{x}} & \sum_{i=1}^{N} x_{i} \\
\text { s.t. } & \sum_{i=1}^{N} \frac{c_{i}}{\alpha_{i} g\left(x_{i}\right)} \leq 1, \\
& x_{i} \in \mathbb{Z}^{+}, \forall i,
\end{array}
$$

where $\boldsymbol{x}=\left[x_{1}, \ldots, x_{N}\right]^{T}$; Constraint (8b) comes from the necessary condition; Constraint $(8 \mathrm{c})$ is the positive integer constraint on the number of sensor nodes to be deployed in each region, which makes the problem non-trivial to solve. However, it is easy to know that Requirement (7) is also the necessary condition that Problem (8) has feasible solution. Then, in the next subsection, we will discuss the solution algorithms to solve Problem (8) when Condition (7) is satisfied.

\section{B. Solution Method}

Here, we will derive an approach to achieve a lower-bound to the required number of sensor nodes by relaxing the integer constraint, and a sub-optimal solution by rounding the relaxed solution. Then, we will provide an approach for the optimal solution. It should be notice that, due to that this is the first work to consider node deployment problem in WET enabled WSNs, the suboptimal approach is for the comparison in the simulation.

1) Relaxation approach: Observing that the integer constraints in Problem (8) make the problem non-trivial, we relax these constraints to non-negative constraints, such that an upper bound solution can be achieved. The relaxed problem is as follows:

$$
\begin{array}{ll}
\min _{\boldsymbol{x}} & \sum_{i=1}^{N} x_{i} \\
\text { s.t. } & \sum_{i=1}^{N} \frac{c_{i}}{\alpha_{i} g\left(x_{i}\right)} \leq 1, \\
& x_{i} \geq 1, \forall i .
\end{array}
$$

Then, the relaxed problem (9) is a convex optimization problem, as can be shown by the following lemma.

Lemma 3: Consider Problem (9) where $c_{i}, \alpha_{i}>0, g^{\prime}\left(x_{i}\right) \geq$ $0, g^{\prime \prime}\left(x_{i}\right) \leq 0, \forall x_{i} \geq 1, g(1)=1$. It is a convex optimization problem.

Proof: The proof can be found in [24].

According to Lemma 3, we can efficiently achieve the optimal solution of Problem (9), denoted by $x_{i}^{\text {re }}$. Observing that $x_{i}$ should be integer, we can thus deploy $\left\lceil x_{i}^{\mathrm{re}}\right\rceil$ at $l_{i}$, such that the WSN is immortal under WET. We say such deployment is based on the relaxation approach. Then, we have the following theorem:

Theorem 1: Consider optimization Problem (8) with optimal solution $f^{*}$. Denote $f^{\text {re }}$ the number of sensor nodes to 


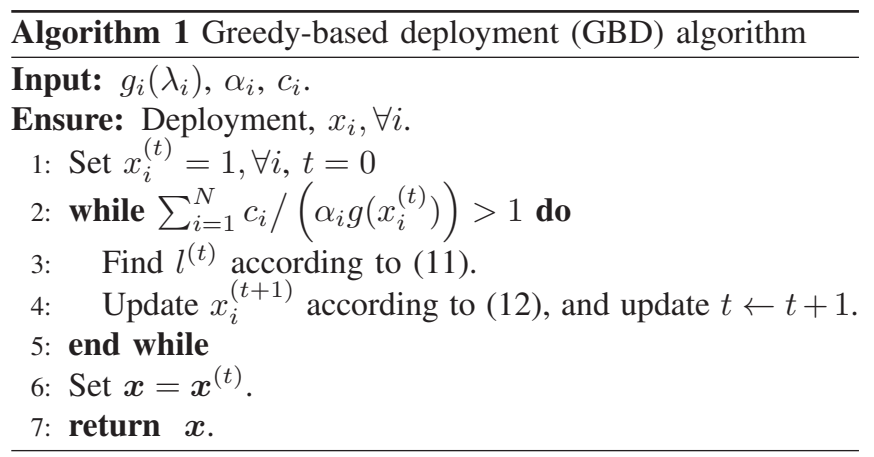

deploy based on the relaxation approach. Then, $f^{*}$ is bounded as

$$
\sum_{i=1}^{N} x_{i}^{\mathrm{re}} \leq f^{*} \leq f^{\mathrm{re}} \triangleq \sum_{i=1}^{N}\left\lceil x_{i}^{\mathrm{re}}\right\rceil,
$$

where $x_{i}^{\text {re }}$ is the optimal solution of Problem (9).

Proof: The proof can be found in [24]

Such proposition provides an upper bound and a lower bound on the total number of nodes to deploy, which will be used in the analysis of the complexity of the algorithms later. Also, we can know that the difference of the upper bound and the lower bound is at most $N$.

2) Optimal deployment: Although Problem (8) is an integer optimization, we will develop an algorithm to find the optimal solution. We will first provide the solution algorithm, and then we show its optimality.

The algorithm is based on greedy improvement, i.e., greedily deploy one sensor node into the field after another, until Constraint (8b) is satisfied. Observing that $x_{i} \in \mathbb{Z}^{+}$, we have that $x_{i} \geq 1, \forall i$. Thus, to begin with, we set $x_{i}^{(0)}=1, \forall i$ initially. In each iteration $t$, if $\boldsymbol{x}^{(t)}=\left[x_{1}^{(t)}, \ldots, x_{n}^{(t)}\right]^{T}$ does not satisfy Constraint (8b), we find the region with the largest benefit to deploy a sensor node, i.e.,

$$
l^{(t)}=\arg \max _{i}\left\{\frac{c_{i}}{\alpha_{i} g\left(x_{i}^{(t)}\right)}-\frac{c_{i}}{\alpha_{i} g\left(x_{i}^{(t)}+1\right)}\right\} .
$$

Then, we update $x_{i}^{(t+1)}$ by

$$
x_{i}^{(t+1)}= \begin{cases}x_{i}^{(t)}+1 & \text { if } i=l^{(t)} \\ x_{i}^{(t)} & \text { otherwise } .\end{cases}
$$

The complete algorithm is shown in Algorithm 1, and the output of Algorithm is denoted by $\boldsymbol{x}^{\mathrm{GBD}}$.

The optimality of the GBD algorithm is as described in the following theorem.

Theorem 2: Consider feasible optimization Problem (8). Then, the GBD algorithm achieves an optimal deployment in terms of the number of deployed sensor nodes. That is, there exists no other deployment $\boldsymbol{x}$, such that $\sum_{i=1}^{N} x_{i}<$ $\sum_{i=1}^{N} x_{i}^{\mathrm{GBD}}$.

To prove this theorem, we first show the following lemma.

Lemma 4: Consider feasible optimization Problem (8). The deployment achieved by the GBD algorithm at the $t$-th iteration is $\boldsymbol{x}^{(t)}=\left[x_{i}^{(t)}, \ldots, x_{N}^{(t)}\right]^{T}$. Then, for any other deployment $\tilde{\boldsymbol{x}}=\left[\tilde{x}_{1}, \ldots, \tilde{x}_{N}\right]^{T}$ such that $\sum_{i=1}^{N} \tilde{x}_{i}=$ $\sum_{i=1}^{N} x_{i}^{(t)}$, we have that

$$
\sum_{i=1}^{N} \frac{c_{i}}{\alpha_{i} g\left(\tilde{x}_{i}\right)} \geq \sum_{i=1}^{N} \frac{c_{i}}{\alpha_{i} g\left(x_{i}^{(t)}\right)} .
$$

Proof: We also define function $F(\boldsymbol{x})=$ $\sum_{i=1}^{N} c_{i} /\left(\alpha_{i} g\left(x_{i}\right)\right)$. Since $\tilde{\boldsymbol{x}} \neq \boldsymbol{x}^{(t)}$ and $\sum_{i=1}^{N} \tilde{x}_{i}=$ $\sum_{i=1}^{N} x_{i}^{(t)}$, we have that there exists an index pair $j$ and $k$, such that $\tilde{x}_{j}<x_{j}^{(t)}$ and $\tilde{x}_{k}>x_{k}^{(t)}$. Then, we first show that, we can modify $\tilde{\boldsymbol{x}}$ to be $\tilde{\boldsymbol{x}}^{\prime}=\left[\tilde{x}_{1}^{\prime}, \ldots, \tilde{x}_{N}^{\prime}\right]^{T}$ by the following operation

$$
\tilde{x}_{i}^{\prime}= \begin{cases}\tilde{x}_{i}+1 & \text { if } i=j \\ \tilde{x}_{i}-1 & \text { if } i=k \\ \tilde{x}_{i} & \text { otherwise }\end{cases}
$$

such that

$$
F(\tilde{\boldsymbol{x}})=\sum_{i=1}^{N} \frac{c_{i}}{\alpha_{i} g\left(\tilde{x}_{i}\right)} \geq \sum_{i=1}^{N} \frac{c_{i}}{\alpha_{i} g\left(\tilde{x}_{i}^{\prime}\right)}=F\left(\tilde{\boldsymbol{x}}^{\prime}\right) .
$$

To show (15), it is equivalent to show

$$
\frac{c_{j}}{\alpha_{j} g\left(\tilde{x}_{j}\right)}+\frac{c_{k}}{\alpha_{k} g\left(\tilde{x}_{k}\right)} \geq \frac{c_{j}}{\alpha_{j} g\left(\tilde{x}_{j}+1\right)}+\frac{c_{k}}{\alpha_{k} g\left(\tilde{x}_{k}-1\right)} \text {. }
$$

Recall Equation (11) that the index of the region with the largest benefit to deploy a sensor node in the $t$-th iteration is $l^{(t)}$. Then, due to that $\tilde{x}_{j}<x_{j}^{(t)}$, we have that

$\frac{c_{j}}{\alpha_{j} g\left(\tilde{x}_{j}\right)}-\frac{c_{j}}{\alpha_{j} g\left(\tilde{x}_{j}+1\right)} \geq \frac{c_{l^{(t)}}}{\alpha_{l^{(t)}} g\left(x_{l^{(t)}}^{(t)}\right)}-\frac{c_{l^{(t)}}}{\alpha_{l^{(t)}} g\left(x_{l^{(t)}}^{(t)}+1\right)}$.

Similarly, as $\tilde{x}_{k}>x_{k}^{(t)}$, we have that

$$
\begin{aligned}
\frac{c_{l^{(t)}}}{\alpha_{l^{(t)}} g\left(x_{l^{(t)}}^{(t)}\right)}-\frac{c_{l^{(t)}}}{\alpha_{l^{(t)}} g\left(x_{l^{(t)}}^{(t)}+1\right)} & \geq \frac{c_{k}}{\alpha_{k} g\left(x_{k}^{(t)}\right)}-\frac{c_{k}}{\alpha_{k} g\left(x_{k}^{(t)}+1\right)} \\
& \geq \frac{c_{k}}{\alpha_{k} g\left(\tilde{x}_{k}-1\right)}-\frac{c_{k}}{\alpha_{k} g\left(\tilde{x}_{k}\right)},
\end{aligned}
$$

where the last inequality holds due to that $\tilde{x}_{k}-1 \geq x_{k}^{(t)}$, $g^{\prime}(x) \geq 0$ and $g^{\prime \prime}(x) \leq 0$. Consequently, we have that

$$
\frac{c_{j}}{\alpha_{j} g\left(\tilde{x}_{j}\right)}-\frac{c_{j}}{\alpha_{j} g\left(\tilde{x}_{j}+1\right)} \geq \frac{c_{k}}{\alpha_{k} g\left(\tilde{x}_{k}-1\right)}-\frac{c_{k}}{\alpha_{k} g\left(\tilde{x}_{k}\right)},
$$

which is equivalent to (16), and thus (15) holds. It means that Operation (14) does not increase $F(\boldsymbol{x})$.

Observing also that Operation (14) does not change the total number of deployed sensor nodes in the field, we have that for any deployment $\tilde{\boldsymbol{x}}$ other than $\boldsymbol{x}^{(t)}$, there exists a sequence of Operation (14) to achieve $\boldsymbol{x}^{(t)}$, such that

$$
\sum_{i=1}^{N} \frac{c_{i}}{\alpha_{i} g\left(\tilde{x}_{i}\right)} \geq \sum_{i=1}^{N} \frac{c_{i}}{\alpha_{i} g\left(x_{i}^{(t)}\right)},
$$

which completes the proof.

Based on Lemma 4, we are now in the position of proving Theorem 2:

Proof of Theorem 2: The proof is based on contradiction. 
Suppose there exists another feasible solution $\tilde{\boldsymbol{x}}$, such that $\sum_{i=1}^{N} \tilde{x}_{i}<\sum_{i=1}^{N} x_{i}^{\mathrm{GBD}}$. Then, we have that

$$
F(\tilde{\boldsymbol{x}})=\sum_{i=1}^{N} \frac{c_{i}}{\alpha_{i} g\left(\tilde{x}_{i}\right)} \leq 1 .
$$

Assume that GBD algorithm takes $t$ iterations to achieve the final deployment $\boldsymbol{x}^{\mathrm{GBD}}$. Denote the deployment achieved by GBD algorithm at the $(t-1)$-th iteration is $\boldsymbol{x}^{(t-1)}$. Then, Line 2 of Algorithm 1 gives us that $F\left(\boldsymbol{x}^{\mathrm{GBD}}\right) \leq 1<F\left(\boldsymbol{x}^{(t-1)}\right)$.

Denote $\left\|\boldsymbol{x}^{(t-1)}\right\|=\sum_{i=1}^{N} x_{i}^{(t-1)}$, and $\|\tilde{\boldsymbol{x}}\|=\sum_{i=1}^{N} \tilde{x}_{i}$. Then $\sum_{i=1}^{N} \tilde{x}_{i}<\sum_{i=1}^{N} x_{i}^{\mathrm{GBD}}$ gives us that $\left\|\boldsymbol{x}^{(t-1)}\right\| \geq\|\tilde{\boldsymbol{x}}\|$. Then, we can construct $\tilde{\tilde{\boldsymbol{x}}}=\left[\tilde{x}_{1}, \ldots, \tilde{x}_{N-1}, \tilde{x}_{N}+\left\|\boldsymbol{x}^{(t-1)}\right\|-\right.$ $\|\tilde{\boldsymbol{x}}\|]^{T}$, such that $\|\tilde{\tilde{\boldsymbol{x}}}\|=\left\|\boldsymbol{x}^{(t-1)}\right\|$, and

$$
\begin{aligned}
F(\tilde{\tilde{\boldsymbol{x}}}) & =\sum_{i=1}^{N-1} \frac{c_{i}}{\alpha_{i} g\left(\tilde{x}_{i}\right)}+\frac{c_{N}}{\alpha_{N} g\left(\tilde{x}_{N}+\left\|\boldsymbol{x}^{(t-1)}\right\|-\|\tilde{\boldsymbol{x}}\|\right)} \\
& \leq \sum_{i=1}^{N} \frac{c_{i}}{\alpha_{i} g\left(\tilde{x}_{i}\right)} \leq 1<F\left(\boldsymbol{x}^{(t-1)}\right),
\end{aligned}
$$

which is contradicted to Lemma 4. As a result, there is no other feasible solutions $\tilde{x}$, such that $\sum_{i=1}^{N} \tilde{x}_{i}<\sum_{i=1}^{N} x_{i}^{\mathrm{GBD}}$, which completes the proof.

Theorem 2 shows that our GBD algorithm can achieve an optimal solution of the deployment Problem (8), and the WSN is immortal for networking purpose. In reality, this deployment is a lower bound of the nodes to deploy for immortality of the WSN. The reason is that issues such as node failures and uncertainty in the parameters should be also considered. Thus, we need to deploy more nodes in each region. It could be one of our future works.

\section{WET Scheduling at BAse Station}

Recalling that Condition (8b) is a necessary condition for the immortality of the WSN, now we are going to show that, when the capacity of the nodes are large enough, such condition is also sufficient. We are going to provide a WET scheduling scheme at the base station that leads to the immortality of the WSN.

We first provide the activation scheme $z_{i j}(t)$ in each region as follows. Recalling that $E_{i j}(t)$ is the residual energy of the $j$-th node in $l_{i}$, we let $z_{i j}(t)=1$ if and only if $j$ is the smallest index such that $E_{i j}(t) \leq E_{i k}(t), \forall k \neq j$. That is, in each timeslot, the node with maximum lifetime is responsible for sensing, data receiving and transmitting, where the node's lifetime of node $v_{i j}$ is defined as $E_{i j}(t) / c_{i}$. We call such activation scheme Max-Activation, as shown in Algorithm 2. Since models of the energy consumption and charging of the nodes in the same region are roughly identical, we can use a virtual node $\tilde{v}_{i}$ to represent the sensor node with minimum residual node's lifetime in $l_{i}$. Then, we have that the residual energy of $\tilde{v}_{i}$ is $E_{i}(t) \triangleq \min \left\{E_{i j}(t)\right\}$. Recalling that, in any timeslot, only the sensor node with maximum node's lifetime among the nodes in the same region consumes energy $c_{i}$, we
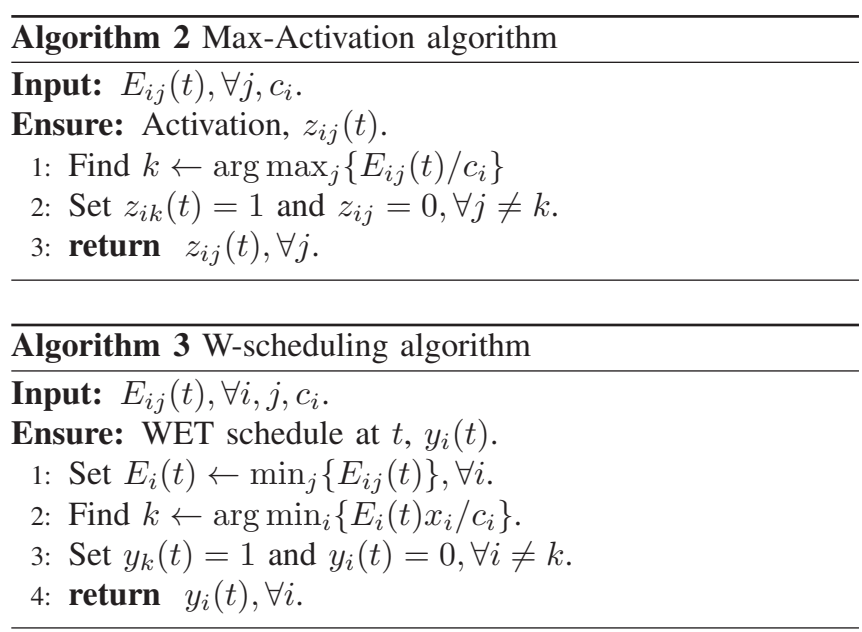

have that the virtual energy consumption $\operatorname{rate}^{2}$ of $\tilde{v}_{i}$ is $c_{i} / x_{i}$. It follows that the minimum node's lifetime of region $l_{i}$ is $E_{i}(t) x_{i} / c_{i}$, and we also call it the lifetime of the region.

Next, we will provide the WET scheduling at the base station. Recall that the network expires when there exists a region where none of the nodes have enough energy to work. Therefore, the WET scheduling tries to transmit energy to the region with the minimum lifetime, which is $\arg \min _{i}\left\{E_{i}(t) x_{i} / c_{i}\right\}$. If there are several regions with the same minimum lifetime, the base station transfers energy to the one with the smallest region's index. Thus, $y_{j}(t)=$ 1 if and only if $j$ is the smallest index that satisfies $j=\arg \min _{i}\left\{E_{i}(t) x_{i} / c_{i}\right\}$, as shown in Algorithm 3. For simplicity, we call such WET scheduling $W$-scheduling.

Then, we are going to show that, under some mild requirement, Condition ( $8 b$ ) is sufficient so that the WSN is immortal, as shown in the following theorem.

Theorem 3: Consider a WSN $(\boldsymbol{L}, \boldsymbol{\lambda}, \boldsymbol{\alpha}, \boldsymbol{x}, \boldsymbol{y}, \boldsymbol{z}, \boldsymbol{E}, B)$. If the WSN satisfies $c_{i} x_{i}+\alpha_{i} \leq E_{i j}(0) \leq B, \forall i, j$, $\sum_{i=1}^{N} c_{i} /\left(\alpha_{i} g\left(x_{i}\right)\right) \leq 1$, and $\boldsymbol{y}, \boldsymbol{z}$ is achieved by W-scheduling algorithm and Max-Activation algorithm respectively, then Condition (8b) is sufficient to make the WSN immortal.

Proof: The proof can be found in [24].

Based on this result, we have the following theorem:

Theorem 4: Consider the Joint Node Deployment and WET Scheduling Problem (4). If the battery capacity of the sensor nodes satisfies $c_{i} x_{i}+\alpha_{i} \leq E_{i j}(0) \leq B, \forall i, j$, then the deployment $\boldsymbol{x}$ achieved by GBD algorithm, the $\boldsymbol{y}$ achieved by $\mathrm{W}$-scheduling, and the $z$ achieved by Max-Activation is one optimal solution of the problem.

Proof: The proof can be found in [24].

As sensor nodes are generally designed for long term monitoring, their battery should be much larger than their energy consumption power, i.e., $B \gg c_{i}$. Consequently, the

\footnotetext{
${ }^{2}$ This corresponds to the case where all the sensor nodes in $l_{i}$ have the same residual energy. Thus, they take turns to activate with period $x_{i}$. Then, in every $x_{i}$ timeslots, the residual energy of all the sensor nodes reduces by $c_{i}$, which means that, averagely the virtual node $\tilde{v}_{i}$ consumes $c_{i} / x_{i}$ in a timeslot.
} 
TABLE I

COMPARISONS OF THE LITERATURES ON WET FOR WSN

\begin{tabular}{ccccc}
\hline Paper & Deployment & $\begin{array}{c}\text { WET } \\
\text { scheduling }\end{array}$ & Other factors & Charger type \\
\hline our work & $\checkmark$ & $\checkmark$ & $\begin{array}{c}\text { sleep-awake } \\
\text { schedule }\end{array}$ & static, single \\
\hline$[7]$ & - & $\checkmark$ & data routing & mobile \\
\hline$[9]$ & - & $\checkmark$ & - & static, single \\
\hline$[21]$ & - & $\checkmark$ & data routing & mobile \\
\hline$[25]$ & Chargers & $\checkmark$ & - & static, multi \\
\hline$[22]$ & Chargers & - & data routing & static, multi \\
\hline
\end{tabular}

requirement of $\alpha_{i}+c_{i} x_{i} \leq B$ generally holds for all $i, j$. We conclude that, the GBD algorithm, the W-scheduling, and the Max-Activation are applicable to efficiently solve the joint node deployment and WET scheduling problem.

\section{COMPARISONS AND SIMULATIONS}

Note that this is the first paper jointly consider node deployment and WET scheduling for WSNs lifetime, we first provide some qualitative comparisons of our approach with other approaches in the literature, as summarized in Table I.

It can be seen that, most of the literatures ([7], [9], [21]) considered the WET problems where the WSN deployment is given, whereas the work in [25] considers the deployment of the WET chargers to cover a certain region. In fact, the deployment of the sensor nodes, which relates to the total received energy and the per-node energy consumption, is also important to network lifetime. Consequently, it should be jointly considered in the WET problems, especially from the system design perspetive. We also argue that, our solution approach may be extended to the cases with mobile chargers as in [7], [21], which could be one of our future works.

Then, we present the results of numerical simulations to illustrate our analysis and discuss the performance of the proposed algorithms. The WSN consists of one base station and $N$ sensor nodes with star topology. The network parameters, which are similar to the setting in [7], are set as follows: the battery of sensor nodes is $10.8 \mathrm{k}$ Joule; the energy consumption of sending a packet of a sensor node is 0.05 Joule; the packet generation rates of the sensor nodes are $\lambda_{i}$ per minutes, which vary from 1 to 15 in different cases; the energy transmission power at the base station is 3 Watts; the WET efficiencies of the sensor nodes, $\alpha_{i}$, are randomly picked from [0.005, 0.03]. $g(x)$ is set to be $g(x)=\left(1-q^{x}\right) /(1-q)$, with $q=1-\max \left\{\alpha_{i}\right\} / 0.9^{3}$. The dynamic of the node's battery follows Eq. (1), where $y_{i}(t)$ is determined by the algorithm used in the base station.

First, we evaluate the probability that the WSNs are immortal under different field size and packet rates, if only one sensor node is deployed at each region. The field size, i.e., the number of region of interest to be monitored, ranges from 1 to 30. For each test case, we examine the immortality of the WSN according to Lemma 1, and the results are as shown in Fig. 2(a). The lines with circle, square, cross, and diamond

\footnotetext{
${ }^{3}$ This corresponds to the case where the benefit of deploying a node in a region with $n-1$ nodes in it is $q^{n}$, where $q$ is determined such that $\lim _{x \rightarrow+\infty} \max \left\{\alpha_{i}\right\} g(x) \leq 0.9$.
}

marks represent the required packet rate of each region, $\lambda$, to be uniform randomly chosen from $[0,5],[5,10],[10,15]$, $[15,20]$ per minute, respectively. It is shown that, even when the packet rate is low, i.e., in average 2.5 packets per minute, the WSNs is hard to be immortal under WET when there are more than 30 regions to monitor and only one node is deployed at each region. The probability of immortality of WSNs drops dramatically when the average packet generation increases, which shows that, the idea of deploying only one node at each region is not scaling with the network size. Thus, for a WSNs of large size, we need to deploy additional nodes in each region, such that the WSNs are immortal.

Next, we evaluate the node deployment algorithms,i.e., GBD algorithm and the relaxation approach, in terms of the number of total sensor nodes to be used. The performance of GBD algorithm (diamond marks), as shown in Fig. 2(b), is compared with that of the relaxation approach (circle marks) and a random deployment approach (square marks), in which we deploy sensor nodes one by one in a region that is independently and randomly chosen, until the network is immortal according to Lemma 1 . We also compare the total number of sensor nodes of the GBD algorithm to the lower bound (green dash line) achieved by the relaxed optimization Problem (9). Fig. 2(b) shows the total number of sensor nodes achieved by different algorithms with different field size $N$, where the required packet rate $\lambda_{i}$ are uniform randomly chosen from $[0,20]$ per minute. It is shown that, the additional needed sensor nodes are increasing with $N$, which dues to the sub-additive behaviour of $g(x)$. Furthermore, we can see that, the required number of sensor nodes achieved by GBD algorithm is approximately $90 \%$ and $70 \%$ to that achieved by the relaxation approach and the random approach, and it is very close to the lower bound, which can be explained by the optimality of the GBD algorithm according to Theorem 2. Also, in average 4 nodes and 7 nodes per region is needed for $N=20$ regions and $N=30$ regions, respectively, such that the WSN is immortal for the networking purpose. This shows that it is cost-effective to deploy multiple nodes in the same region to receive enough energy for sensing and data uploading.

Last, we examine that, if the battery buffer sizes of the sensor nodes satisfy the requirement in Theorem 3, the WSN is immortal with the use of GBD algrotihm to deploy the nodes, the use of W-scheduling for WET at the base station side, and Max-Activation scheme described in Section V. For a WSN deployment given by GBD algorithm, we determine the WET $y_{i}(t)$ and the activation $z_{i j}(t)$, and update the nodes' residual energy based on (1). In each timeslot $t$, we find the sensor node with the minimal percentage of residual energy, i.e., $E_{i j}(t) / B$, and denote it by the minimal percentage of residual energy among all the sensor nodes at that timeslot. Fig. 2(c) shows an example of the dynamic of the minimal percentage of residual energy among all the sensor nodes (blue solid line), and the dynamic of the residual energy of a sensor node in region $l_{1}$ (red dash line). We can see that, none of the sensor node depletes its battery, and the minimum percentage of residual 


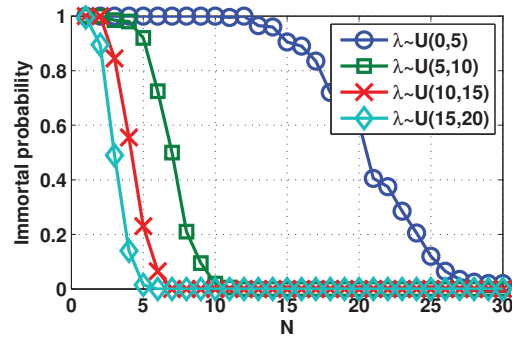

(a)

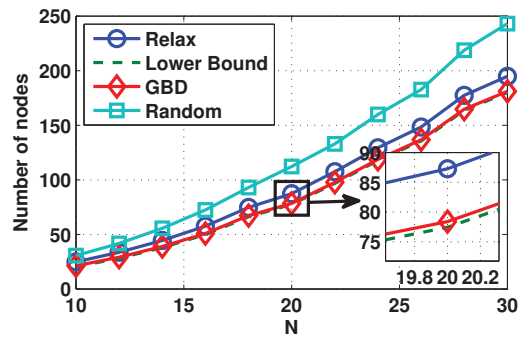

(b)

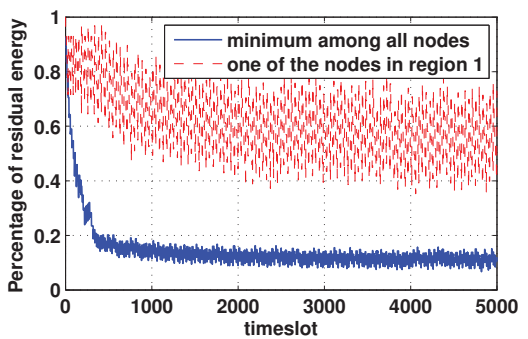

(c)

Fig. 2. (a) The probability of wireless energy transfer enabled sensor networks to be immortal with different field size $N$ and packet rates $\lambda$; (b) Comparison of the required number of sensor nodes achieved by different algorithms; (c) The dynamic of the minimum percentage of residual energy

energy remains at approximate $15 \%$. We conclude that, under the node deployment by GBD algorithm, W-scheduling at the base station side, and Max-Activaition scheme in each region, the WSNs are immortal.

\section{CONCLUSIONS AND FUTURE WORKS}

In this paper, we investigated the joint problem of deployment of sensor nodes and the scheduling the wireless energy transfer in wireless sensor networks, such that the network lifetime is infinite. We studied the necessary condition on the deployment of sensor nodes to make the network immortal. Then, we proposed an algorithm to achieve the optimal node deployment. Finally, we proposed an energy transmission scheme at the base station side and the sensor node activation scheme at the sensor node side. We showed that the deployment and the WET scheduling can lead to the immortality of the WSN if the sensor nodes have enough initial energy. The simulation results showed the effectiveness of the proposed algorithm.

In the future, the study will be extended to the case where the charging efficiency is time-varying due to the randomness of the channel. We are also interested in considering the case with mobile wireless charger.

\section{REFERENCES}

[1] L. Mo, Y. He, Y. Liu, J. Zhao, S.-J. Tang, X.-Y. Li, and G. Dai, "Canopy closure estimates with greenorbs: sustainable sensing in the forest," in Proc. ACM Conference on Embedded Networked Sensor Systems, 2009, pp. 99-112.

[2] R. Du, L. Gkatzikis, C. Fischione, and M. Xiao, "Energy efficient sensor activation for water distribution network based on compressive sensing," IEEE Journal on Selected Areas in Communications, vol. 33, no. 12, pp. 2997-3010, 2015.

[3] Q. Wang, Y. Zhu, and L. Cheng, "Reprogramming wireless sensor networks: challenges and approaches," IEEE Network, vol. 20, no. 3, pp. 48-55, 2006.

[4] J. Taneja, J. Jeong, and D. Culler, "Design, modeling, and capacity planning for micro-solar power sensor networks," in Proceedings of the IEEE international conference on Information processing in sensor networks, 2008, pp. 407-418.

[5] C. Park and P. H. Chou, "Ambimax: Autonomous energy harvesting platform for multi-supply wireless sensor nodes," in Annual IEEE Communications Society on Sensor and Ad Hoc Communications and Networks, vol. 1, 2006, pp. 168-177.

[6] Ö. Zorlu, E. T. Topal, and H. Külah, "A vibration-based electromagnetic energy harvester using mechanical frequency up-conversion method," IEEE Sensors Journal, vol. 11, no. 2, pp. 481-488, 2011.

[7] Z. Li, Y. Peng, W. Zhang, and D. Qiao, "J-roc: a joint routing and charging scheme to prolong sensor network lifetime," in IEEE International Conference on Network Protocols, 2011, pp. 373-382.
[8] S. Bi, C. K. Ho, and R. Zhang, "Wireless powered communication: opportunities and challenges," IEEE Communications Magazine, vol. 53, no. 4 , pp. $117-125,2015$.

[9] R. Du, C. Fischione, and M. Xiao, "Lifetime maximization for sensor networks with wireless energy transfer," in Proc. IEEE International Conference on Communications, 2016, pp. 20-25.

[10] S. Sudevalayam and P. Kulkarni, "Energy harvesting sensor nodes: Survey and implications," IEEE Communications Surveys \& Tutorials, vol. 13, no. 3, pp. 443-461, 2011.

[11] N. Michelusi, K. Stamatiou, and M. Zorzi, "On optimal transmission policies for energy harvesting devices," in Proc. IEEE Information Theory and Applications Workshop, 2012, pp. 249-254.

[12] N. Michelusi and M. Zorzi, "Optimal adaptive random multiaccess in energy harvesting wireless sensor networks," IEEE Trans. on Communications, vol. 63, no. 4, pp. 1355-1372, 2015.

[13] J. Yang, X. Wu, and J. Wu, "Optimal scheduling of collaborative sensing in energy harvesting sensor networks," IEEE Journal on Selected Areas in Communications, vol. 33, no. 3, pp. 512-523, 2015.

[14] D. Mishra and S. De, "Optimal power allocation and relay placement for wireless information and rf power transfer," in Proc. IEEE International Conference on Communications, 2016, pp. 2820-2825.

[15] A. Ozcelikkale, T. McKelvey, and M. Viberg, "Performance bounds for remote estimation with an energy harvesting sensor," in Proc. IEEE International Symposium on Information Theory, 2016.

[16] O. Ozel, K. Tutuncuoglu, J. Yang, S. Ulukus, and A. Yener, "Transmission with energy harvesting nodes in fading wireless channels: Optimal policies," IEEE Journal on Selected Areas in Communications, vol. 29, no. 8, pp. 1732-1743, 2011.

[17] L. Xie, Y. Shi, Y. T. Hou, and A. Lou, "Wireless power transfer and applications to sensor networks," IEEE Wireless Communications, vol. 20, no. 4, pp. 140-145, 2013.

[18] X. Zhou, R. Zhang, and C. K. Ho, "Wireless information and power transfer: Architecture design and rate-energy tradeoff," IEEE Trans. on Communications, vol. 61, no. 11, pp. 4754-4767, 2013.

[19] T.-Q. Wu and H.-C. Yang, "On the performance of overlaid wireless sensor transmission with rf energy harvesting," IEEE Journal on Selected Areas in Communications, vol. 33, no. 8, pp. 1693-1705, Aug 2015.

[20] K. Singh, M.-L. Ku, and J.-C. Lin, "Joint power control and energy transfer for energy harvesting relay networks," in Proc. IEEE International Conference on Communications, 2016, pp. 526-531.

[21] L. Xie, Y. Shi, Y. T. Hou, and H. D. Sherali, "Making sensor networks immortal: An energy-renewal approach with wireless power transfer," IEEE/ACM Trans. on Networking, vol. 20, no. 6, pp. 1748-1761, 2012

[22] T. He, K.-W. Chin, and S. Soh, "On wireless power transfer and max flow in rechargeable wireless sensor networks," IEEE Access, vol. 4, pp. 4155-4167, 2016.

[23] R. Szewczyk, E. Osterweil, J. Polastre, M. Hamilton, A. Mainwaring, and D. Estrin, "Habitat monitoring with sensor networks," Communications of the ACM, vol. 47, no. 6, pp. 34-40, 2004.

[24] "Extended version of paper "joint node deployment and wireless energy transfer scheduling for immortal sensor networks." [Online]. Available: http://tinyurl.com/ju45h5n

[25] M. R. V. Moghadam and R. Zhang, "Node placement and distributed magnetic beamforming optimization for wireless power transfer," arXiv preprint arXiv:1608.00304, 2016. 